تأثير نوعية مياه الري على تحرر البوتاسيوم في بعض الترب الكلسية في محافظة نينوى

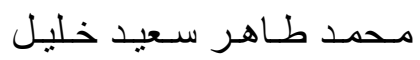

قسم علوم التربة و الموارد المائية / كلية الزر اعة و الغابات / جامعة الموصل ـ العراق

E-mail: dr_mohammed1951@yahoo.com

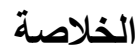

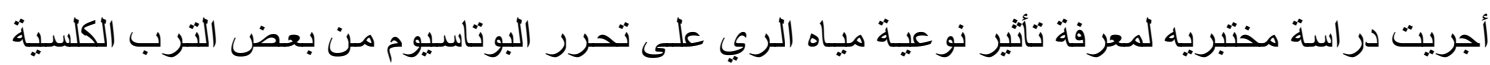

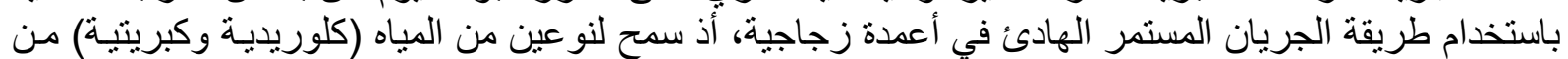

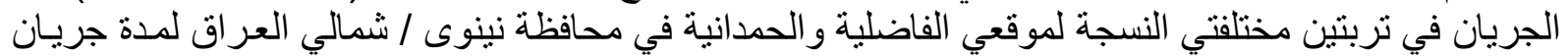

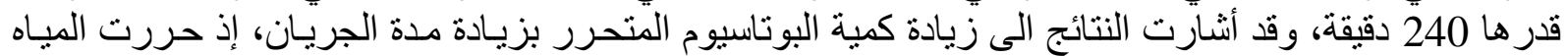

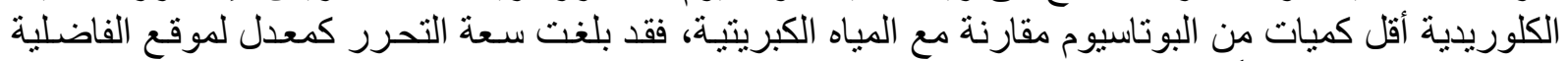

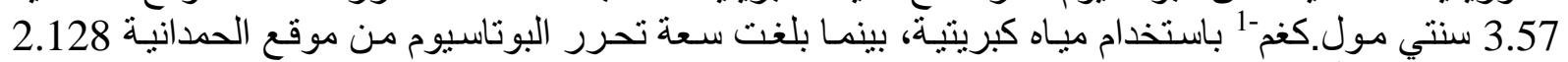

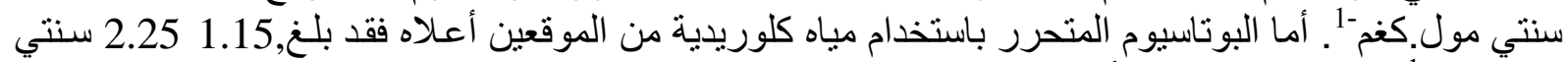

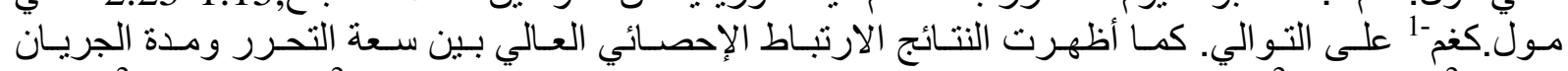

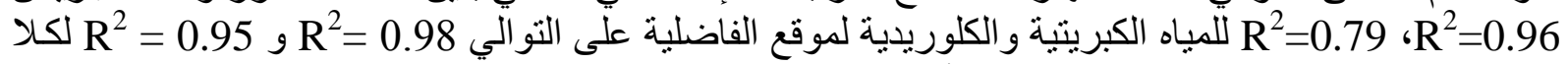

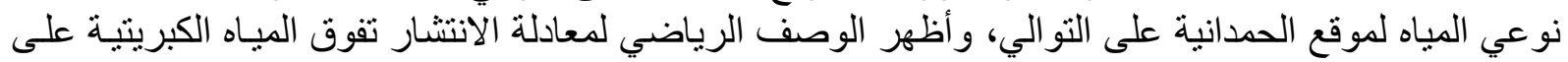

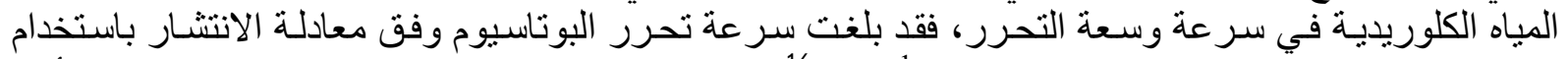

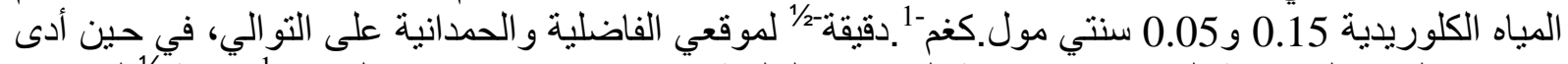

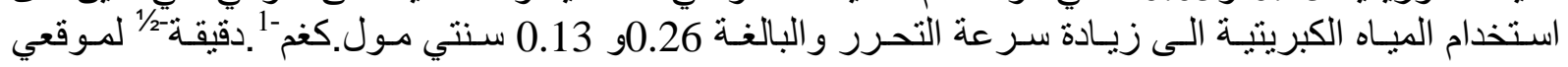
الفاضلية و الحمدانية على التوالي. الكماتئ.

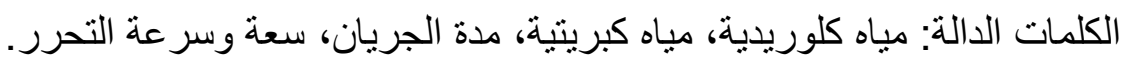
تاريخ تسلم البحث: 2013/1/7 ، وقبوله: 2013/5/6.

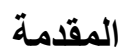

يعد البوتاسيوم عنصر ا غذائيا رئيسيا في حياة النبات ويعاني هذا العنصر من مشـاكل كثيرة في الترب التربه

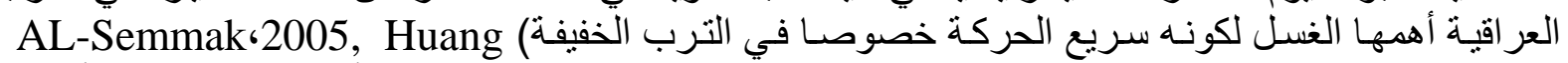

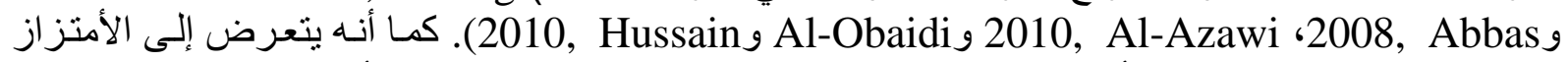

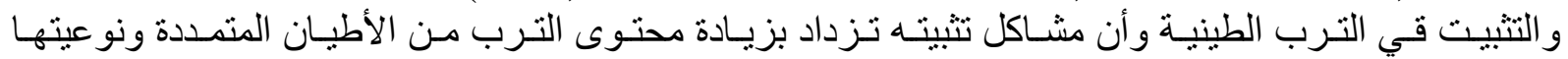
2006, Dahiya و و وآخرون , Shadraoui) و و و و Poonia) Nicderbudde)، ولقد اتجهت الدراسات الحديثة إلى استخدام أساليب عديدة من أجل تحرر

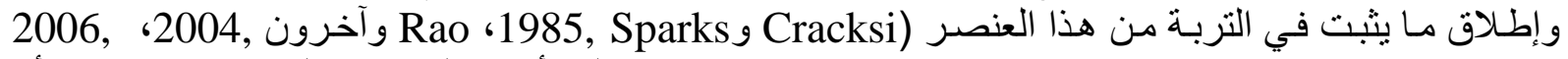

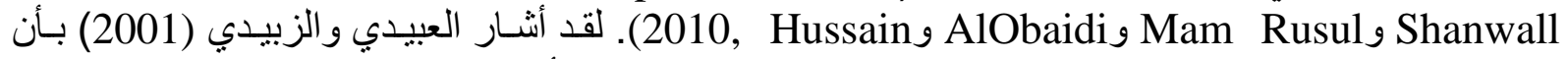

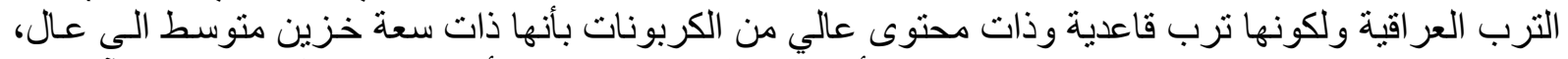

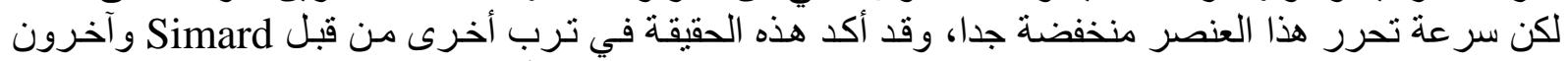

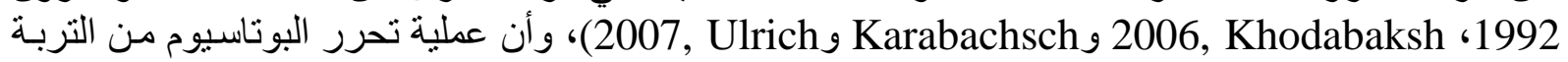

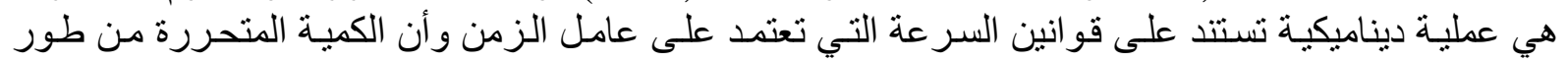

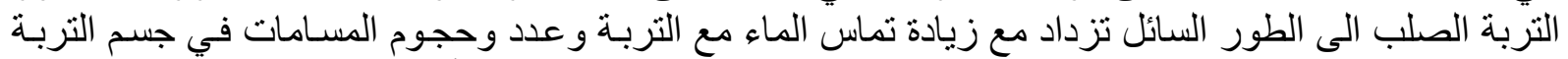

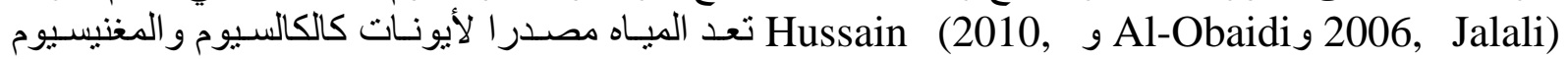

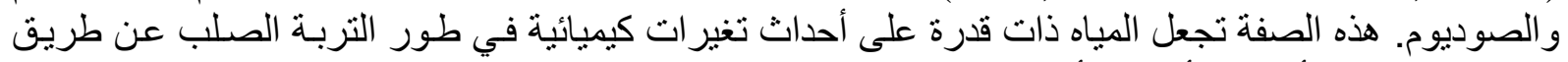

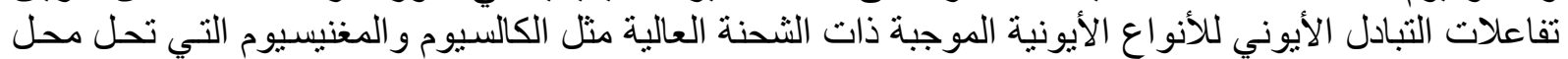

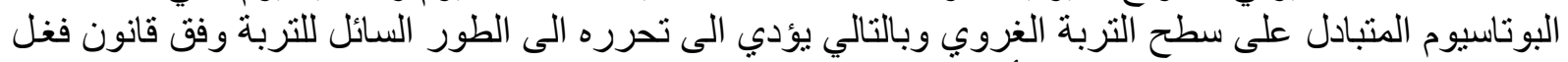

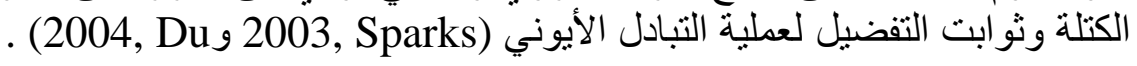

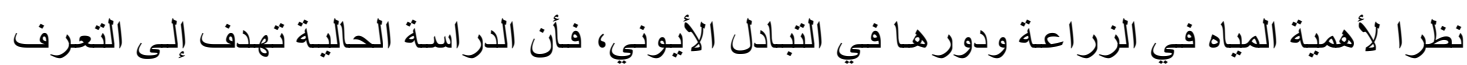

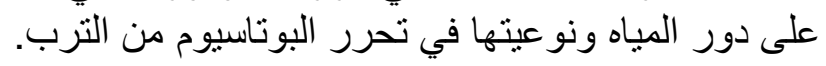




\section{مواد البحث وطر ائقه}

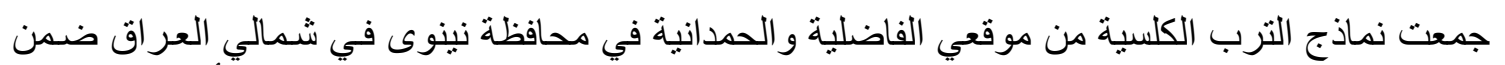

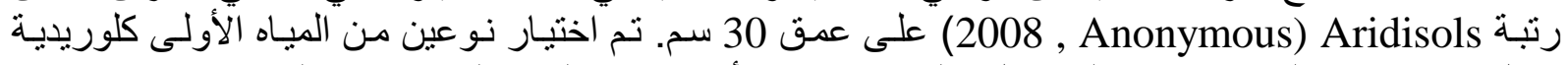

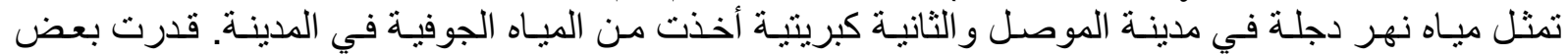

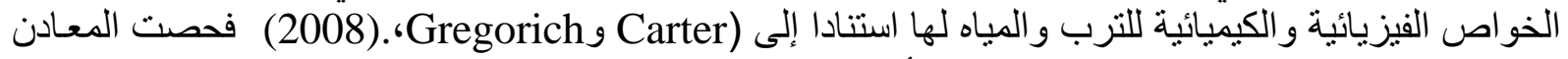

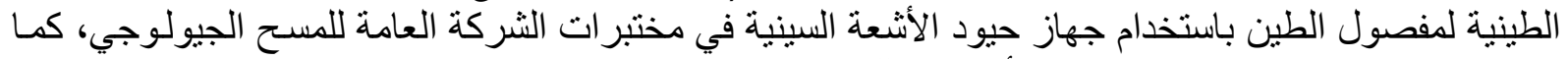

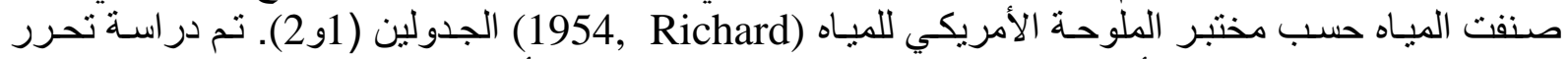

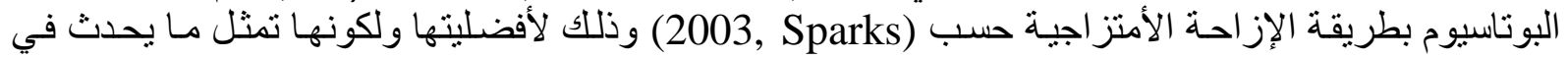

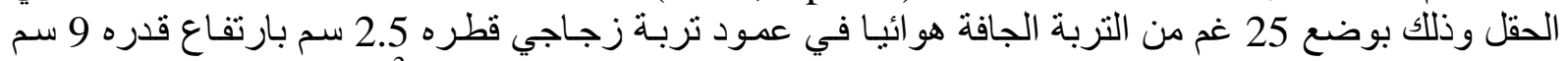

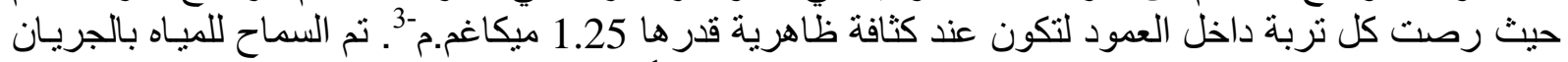

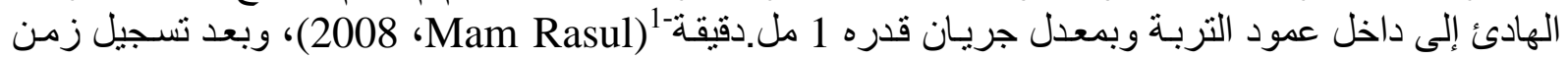

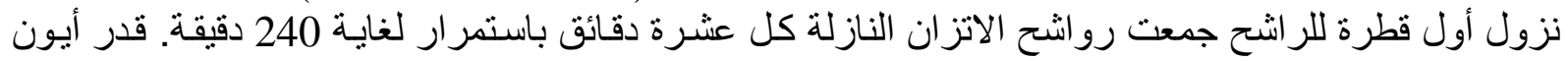

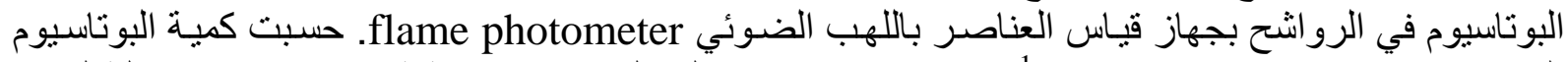

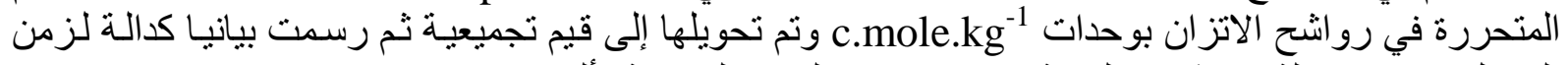

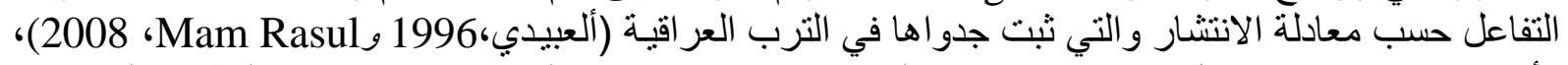

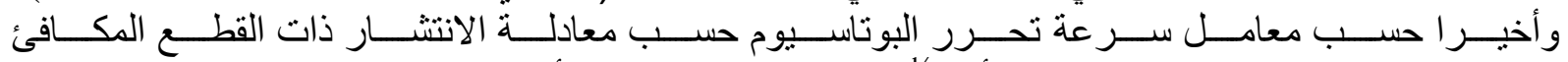
parabolic diffusion

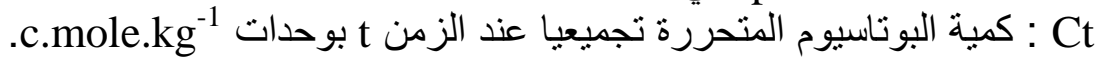
Co (1982), Sparks : ميل العلاقة الإحصائية والذي يعبر عن معامل سرعة الانتشار الظاهري استنادا إلى : K 'Recheigl 2 : الجذر ألتربيعي للزمن.

الجدول (1): بعض الخصائص الفيزيائية والكيميائية والمعدنية لترب الدراسة.

Table (1): Some physical and chemical characteristics of studied soils.

\begin{tabular}{|c|c|c|c|c|}
\hline \multicolumn{2}{|c|}{ Location الموقع } & \multirow{2}{*}{$\begin{array}{l}\text { الوحدة } \\
\text { Unit }\end{array}$} & \multirow{2}{*}{\multicolumn{2}{|c|}{$\begin{array}{c}\text { الصفة } \\
\text { Characteristic }\end{array}$}} \\
\hline Hamdania الحمدانية & Fadhlia الفاضلية & & & \\
\hline 373 & 375 & \multirow{5}{*}{ gm. $\mathrm{kg}^{-1}$} & \multicolumn{2}{|c|}{ clay } \\
\hline 384 & 387 & & \multicolumn{2}{|c|}{ silt } \\
\hline 243 & 238 & & \multicolumn{2}{|c|}{ sand } \\
\hline 210 & 188 & & \multicolumn{2}{|c|}{$\mathrm{CaCO}_{3}$} \\
\hline 13.5 & 14 & & \multicolumn{2}{|c|}{ Organic matter } \\
\hline 0.5 & 0.3 & $\mathrm{dS} . \mathrm{m}^{-1}$ & \multicolumn{2}{|c|}{$\mathrm{EC}$} \\
\hline 7.3 & 7.2 & & \multicolumn{2}{|c|}{$\mathrm{pH}$} \\
\hline 18.7 & 18.9 & c.mole.kg ${ }^{-1}$ & \multicolumn{2}{|c|}{ CEC } \\
\hline 2.4 & 2.6 & \multirow{7}{*}{$\begin{array}{l}\text { m.mole. } \\
\text { liter }\end{array}$} & $\mathrm{Ca}^{+2}$ & \multirow{7}{*}{$\begin{array}{c}\text { Soluble } \\
\text { ions }\end{array}$} \\
\hline 0.8 & 1.1 & & $\mathrm{Mg}^{+2}$ & \\
\hline 0.45 & 0.87 & & $\mathrm{Na}^{+}$ & \\
\hline 0.06 & 0.11 & & $\mathrm{~K}^{+}$ & \\
\hline 1.9 & 2.2 & & $\mathrm{HCO}_{3}^{-}$ & \\
\hline 0.15 & 0.12 & & $\mathrm{Cl}^{-}$ & \\
\hline 1.46 & 2.36 & & $\mathrm{SO}_{4}^{-2}$ & \\
\hline Dominated & Dominated & Smictite & \multirow{4}{*}{\multicolumn{2}{|c|}{ Clay minerals }} \\
\hline Main & Main & Illite & & \\
\hline Rare & Little & Chlorite & & \\
\hline Rare & Rare & Badillite & & \\
\hline
\end{tabular}


الجدول (2) : بعض الخصائص الفيزيائية و الكيميائية لمياه الري المستخدمة.

Table (2): Some physical and chemical characteristics of irrigation water used.

\begin{tabular}{|c|c|c|c|c|c|c|c|c|c|c|c|}
\hline \multirow{3}{*}{$\begin{array}{c}\text { تصنيف الماء } \\
\text { Water } \\
\text { classification }\end{array}$} & \multirow{3}{*}{ SAR } & \multicolumn{7}{|c|}{ Soluble ions الأيونات الذائبة } & \multirow{3}{*}{$\begin{array}{c}\mathrm{EC} \\
\mathrm{dS} \cdot \mathrm{m}^{-}\end{array}$} & \multirow{3}{*}{$\mathrm{pH}$} & \multirow{3}{*}{$\begin{array}{c}\text { نوع الماء } \\
\text { Water } \\
\text { quality }\end{array}$} \\
\hline & & $\mathrm{SO}_{4}^{-2}$ & $\mathrm{Cl}^{-}$ & $\mathrm{HCO}_{3}$ & $\mathrm{~K}^{+}$ & $\mathrm{Na}^{+}$ & $\mathrm{Mg}^{+2}$ & $\mathrm{Ca}^{+2}$ & & & \\
\hline & & \multicolumn{7}{|c|}{ m.mole.liter ${ }^{-1}$} & & & \\
\hline $\mathrm{C}_{2} \mathrm{~S}_{1}$ & 0.4 & 40.3 & 50.5 & 3.2 & 1.8 & 37 & 20 & 35 & 8.02 & 7.8 & sulfuric \\
\hline $\mathrm{C}_{4} \mathrm{~S}_{1}$ & 7.0 & 0.86 & 0.55 & 3.0 & 0.05 & 0.6 & 2.0 & 2.0 & 0.45 & 8.0 & chlorine \\
\hline
\end{tabular}

\section{النتائج والمناقشة}

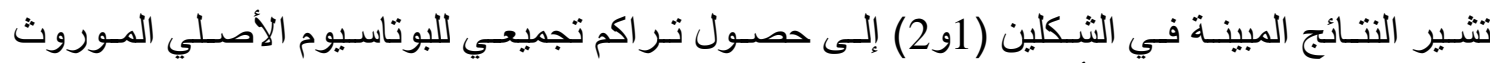
Indigenous

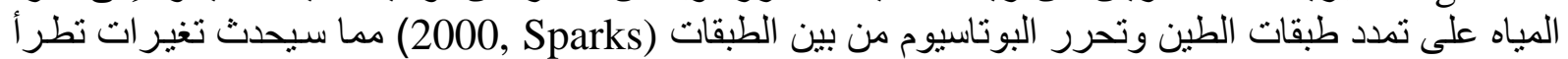

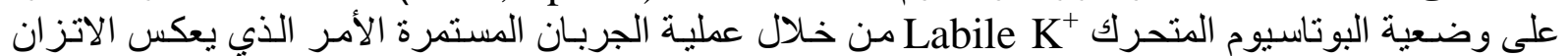

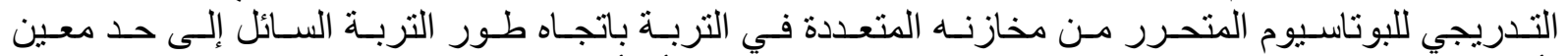

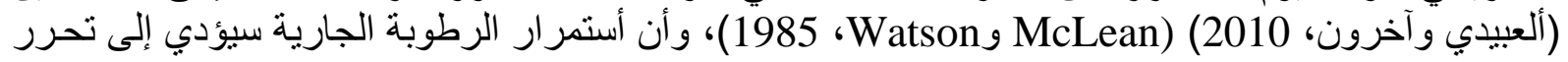

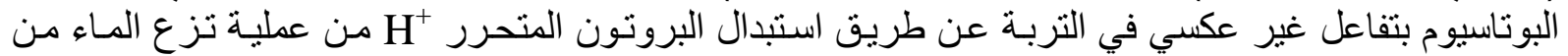

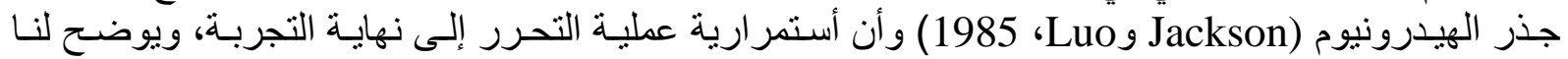

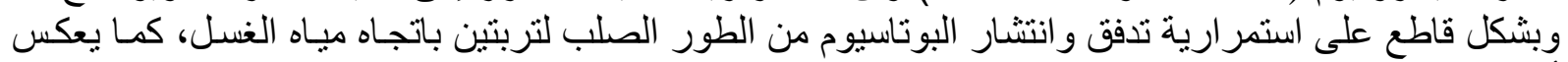

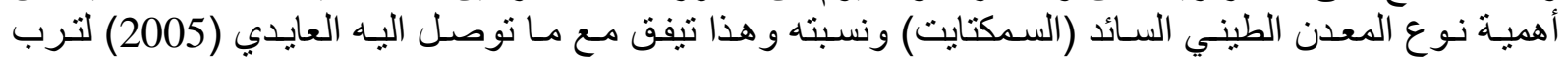

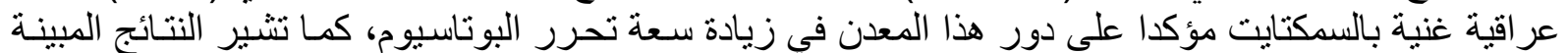

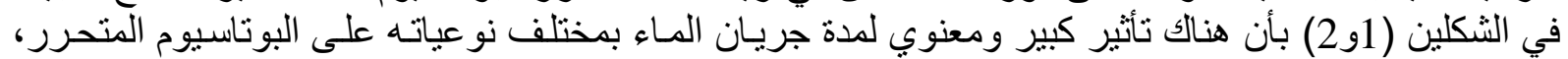

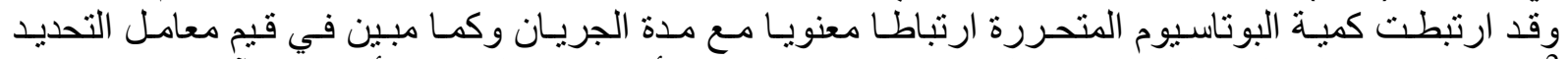

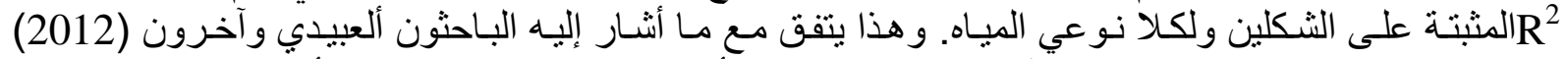

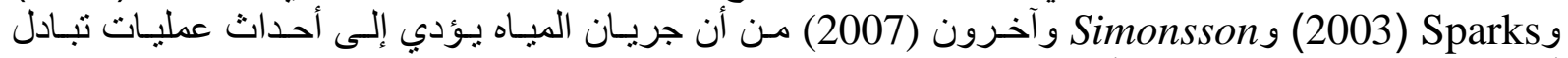

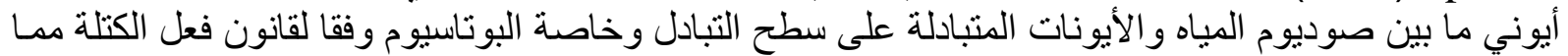

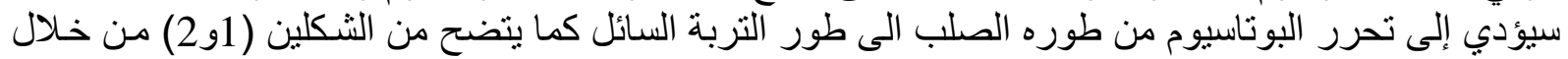

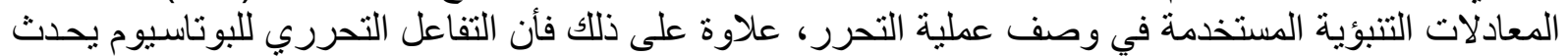

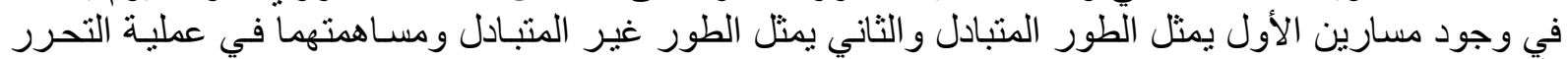
(ألعبيدي، 1996).

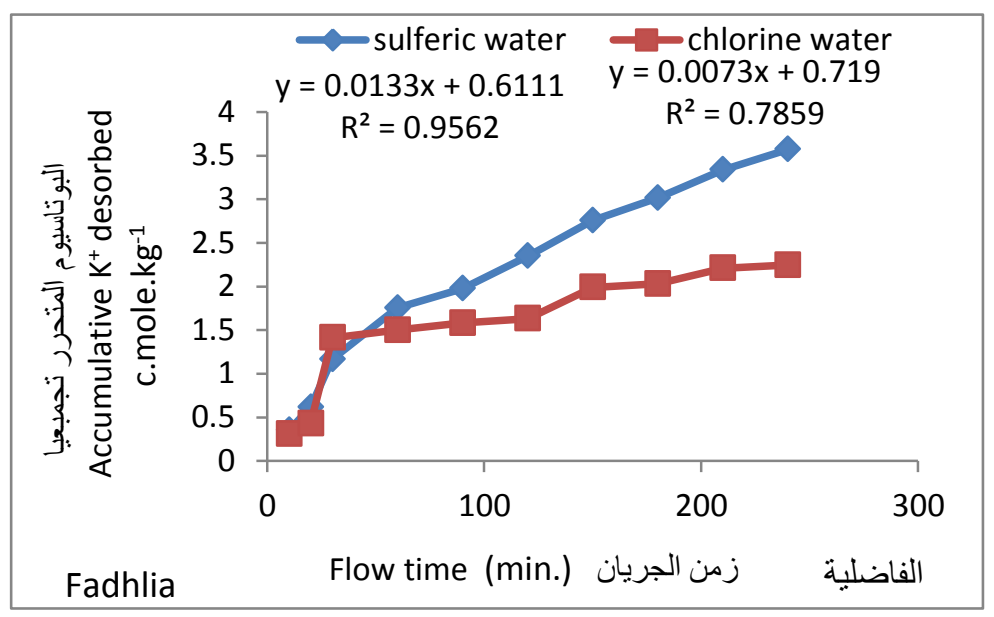

الثكل (1): نأثير نوعية المياه وزمن جريانها على تحرر البوتاسيوم في تربة الفاضلية.

Fig. (1): Effect of water quality and flow time on $\mathrm{K}^{+}$desorption in Fadhlia soil. 


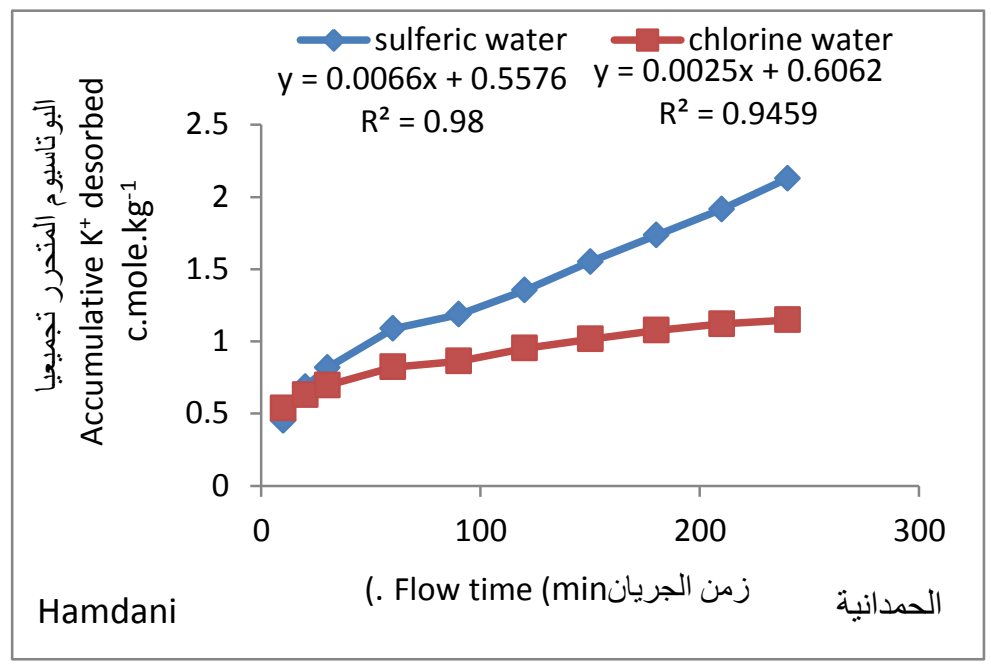

الثكل (2): تأثثر نوعية المياه وزمن جريانها على تحرر البوناسيوم في تربة الحمدانية. Fig. (2) : Effect of water quality and flow time on $\mathrm{K}^{+}$desorption in Hamdania soil.

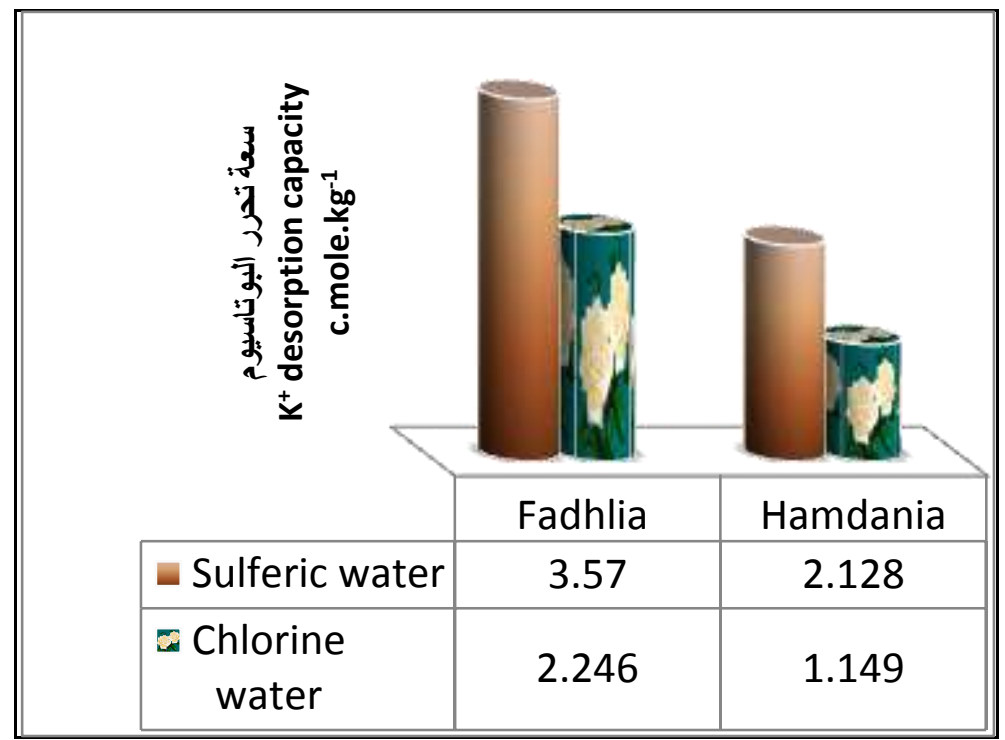

الثكل (3) : تأثثر نوعية المياه على سعة تحرر البوتاسيوم في ترب الدراسة.

Fig. (3): Effect of water quality on $\mathrm{K}^{+}$desorption capacity in investigated soils.

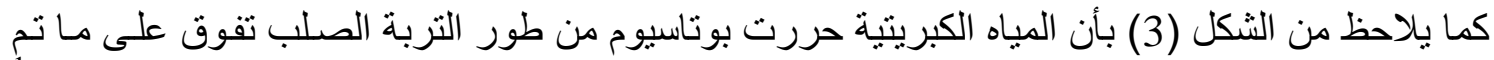

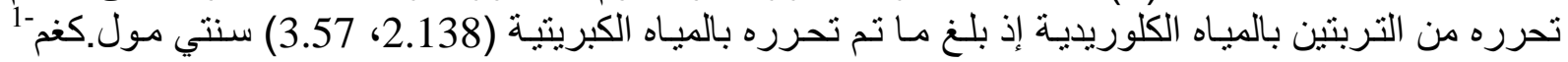

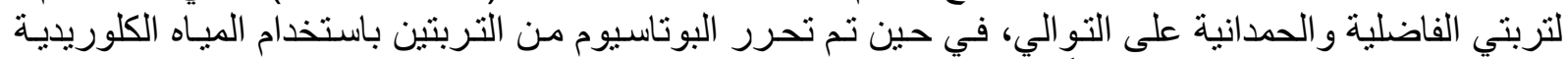

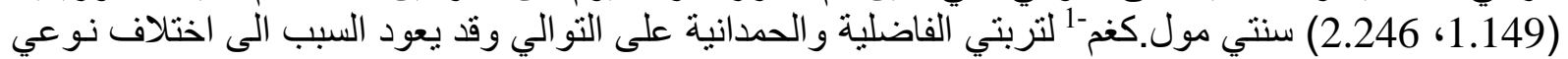

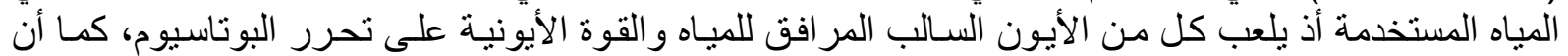

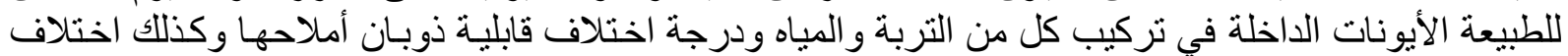

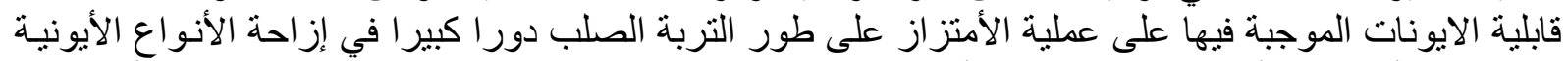

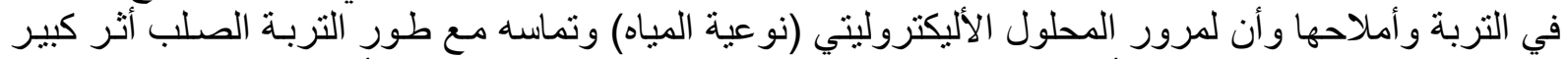

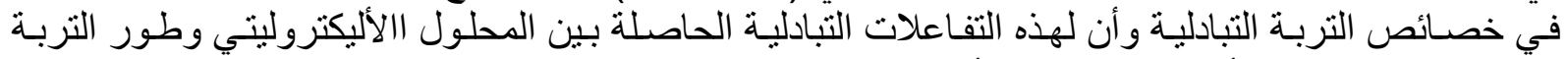

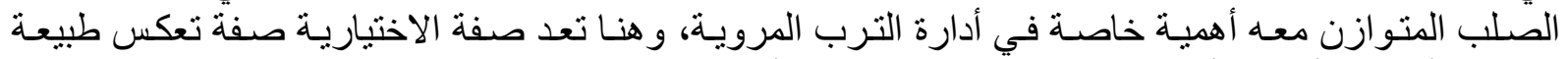

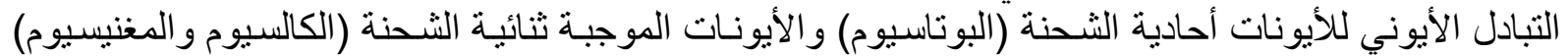

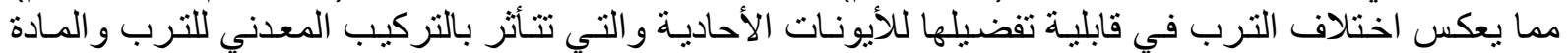


العضوية والسعة التبادلية للأيونات الموجبـة (2007, Jalali Kolahchi) كما تشير النتائج إلى تفوق الميـاه

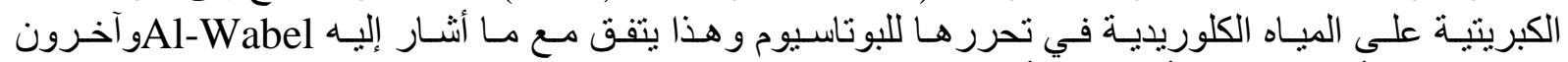

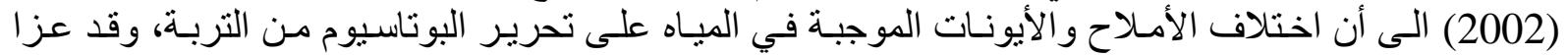

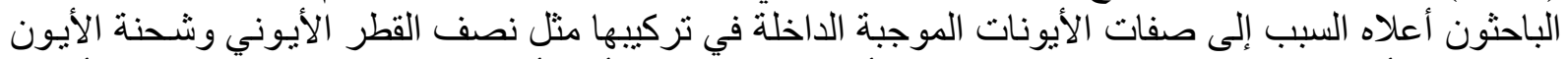

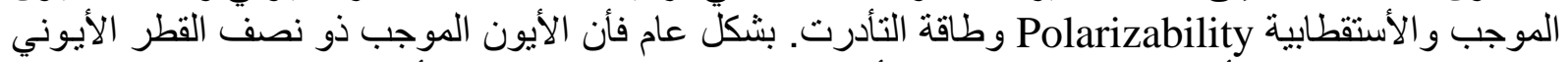

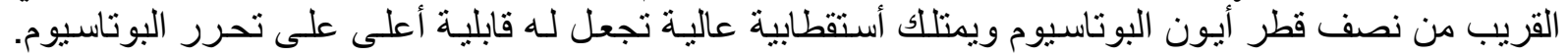

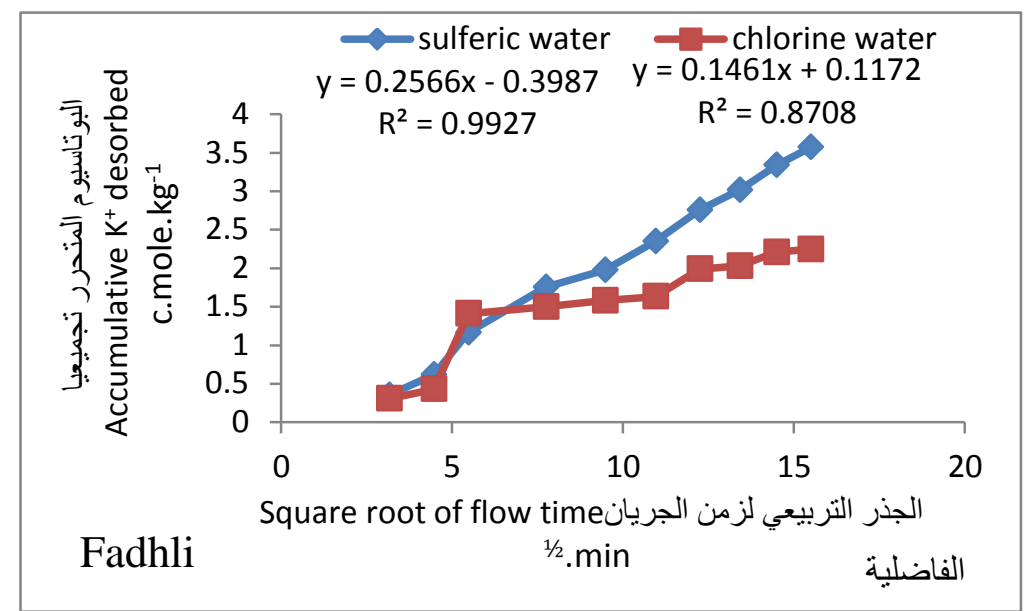

الثكل (4): العلاقة بين الجذر ألتربيعي لزمن الجريان والبوتاسيوم المتحرر تجميعيا في تربة الفاضلية. Fig. (4): Relationship between square root of flow time and accumulative $\mathrm{K}^{+}$desorbed in Fadhlia soil.

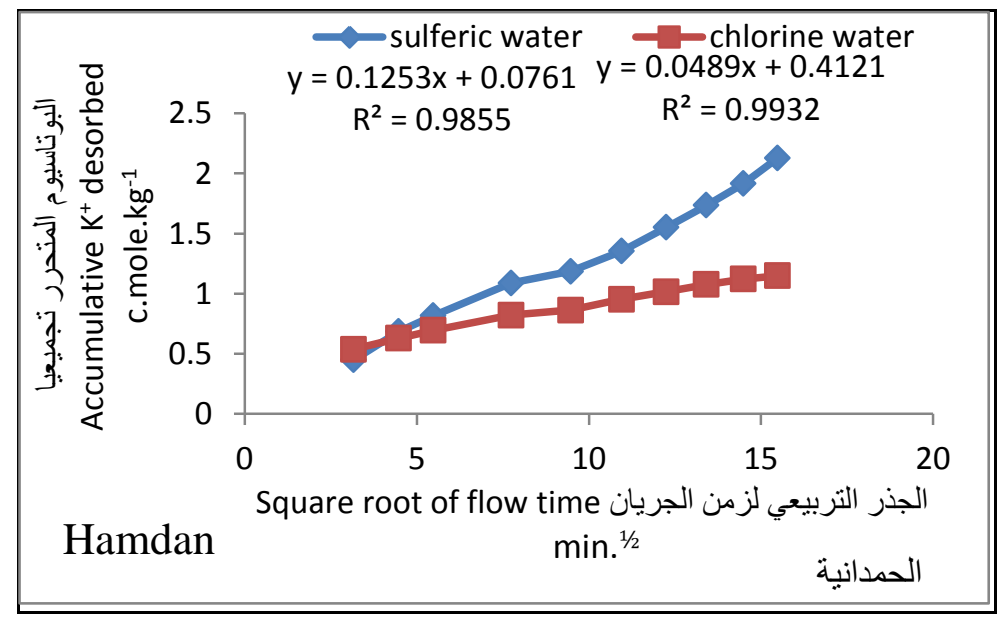

الثكل (5): العلاقة بين الجذر ألتربيعي لزمن الجريان والبوتاسيوم المتحرر تجميعيا في تربة الحمدانية. Fig. (5): Relationship between square root of flow time and accumulative $\mathrm{K}^{+}$desorbed in Hamdania soil.

وفي هذا الصدد وجد Bohn و آخرون (2005) وWang و آخرون (2004) بـأن هناك زيادة في تحرر

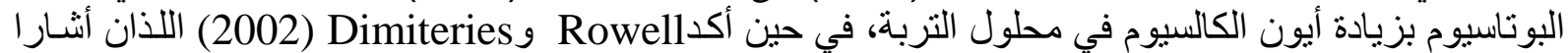

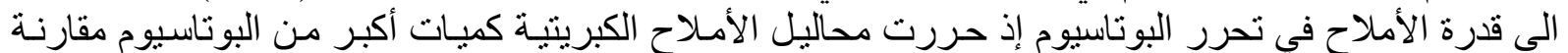

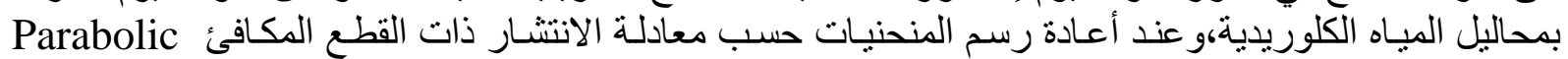
diffusion

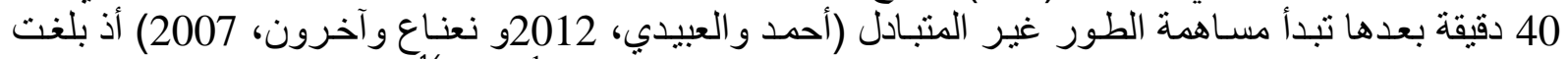

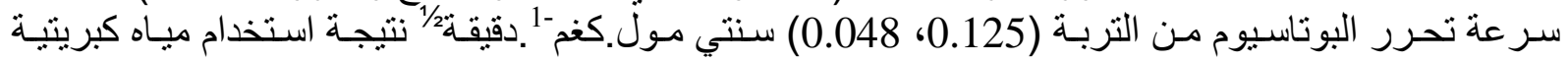


وكلوريدية. أما في موقع الفاضلية ظهر نفس السلوك التحرري مـع تفوق للميـاه الكبريتية على الميـاه الكلوريديـة،

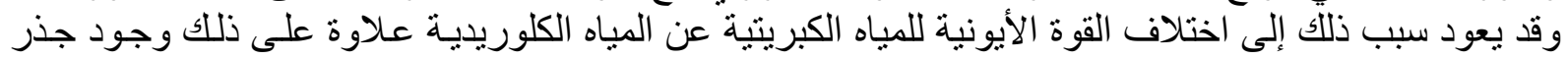
الكبريتات وقدرته المتفوقة في التأثير على مكونات التربة الصلبة مما لإناه

يساهم في سرعة وسعة التحرر (السماك، 2007). يتضح مما ورد أعلاه أهمية استخدام الميـاه الكبريتية

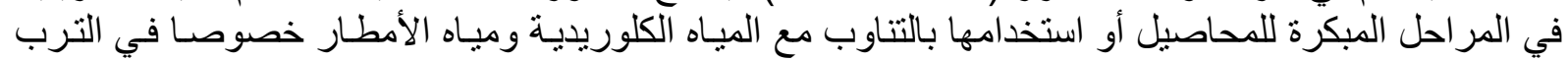
الديمية لرفع جاهزية البوتاسيوم فيها.

\title{
EFFECT OF WATER IRRIGATION QUALITY ON POTASSIUM DESORPTION IN SOME CALCAREOUS SOILS IN NINEVH PROVINCE
}

\author{
Mohammed Tahir Said Khalil \\ Soil Science and Water Resources Dept., College of Agriculture and Forestry, \\ Mosul University. Iraq \\ E-mail: dr_mohammed1951@yahoo.com
}

\begin{abstract}
A laboratory study was conducted to determine the effect of irrigation water quality on potassium desorption in some calcareous soils. It was constructed by miscible displacement technique using chlorine and sulfuric water via two different textured soils from Fadhlia and Hamdania locations in Nineveh province / northern Iraq for 240 minute with 10 minutes interval at a rate flow 1 ml.minute ${ }^{-1}$. Results showed higher values of desorbed potassium rate in sulfuric water which were 3.57, 2.128 c.mole. $\mathrm{kg}^{-1}$, while these rates referred to be much lower than that resulted by using chlorine water $2.25,1.15$ c.mole. $\mathrm{kg}^{-1}$ for both locations respectively. Also high correlation appeared between $\mathrm{K}^{+}$desorption capacity and flow time $\left(\mathrm{R}^{2}=0.96, \mathrm{R}^{2}=\right.$ $0.79)$ for both water qualities in Fadhlia location, while these rates were $\left(R^{2}=0.98, R^{2}\right.$ $=0.95$ ) for Hamdania location respectively. Mathematical description for diffusion equation appeared to be of higher values of $\mathrm{K}^{+}$desorption capacity rates for sulfuric water which were $0.26,0.13 \mathrm{c}$.mole. $\mathrm{kg}^{-1} \cdot \mathrm{min}^{-1 / 2}$ for Fadhlia and Hamdania locations respectively, while these rates for chlorine water were $0.146,0.048$ c.mole. $\mathrm{kg}^{-1} \mathrm{~min} .^{-1 / 2}$ for both locations respectively.
\end{abstract}

Keywords: chlorine water, sulfuric water, Flow time, desorption rate \& capacity.

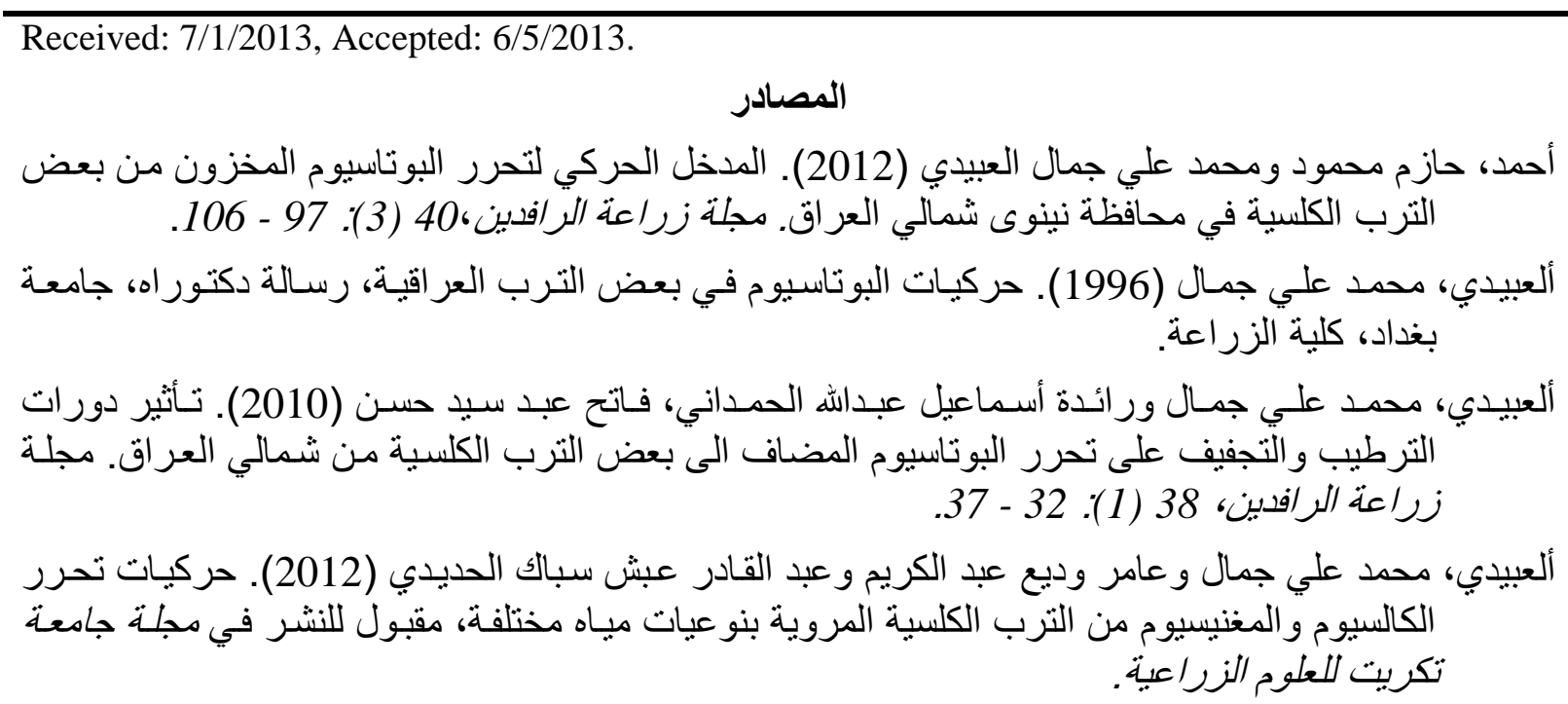


السماك، قيس حسين عبـاس (2007). سلوكية بعض الأسمدة الفوسفاتية في تربـة صـحر اوية مستغلة زر اعيا

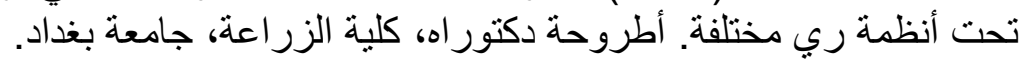

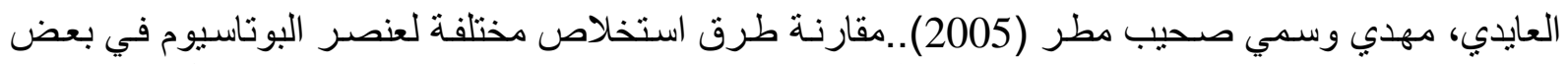

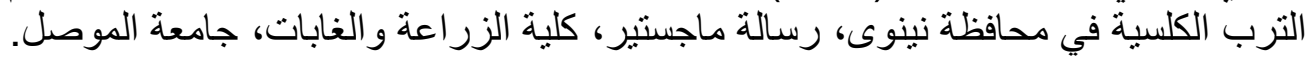

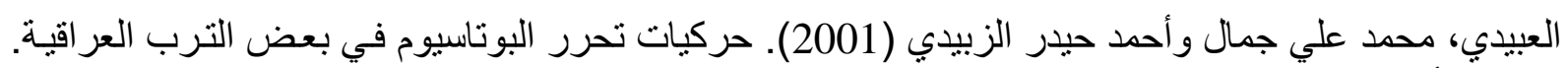

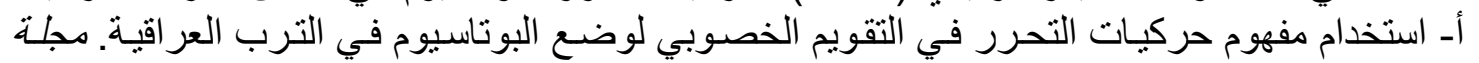
زراعة الرافدين، 32 (2): 33- 40.

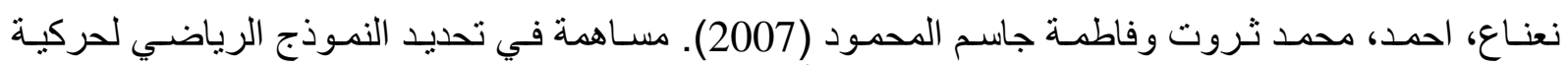

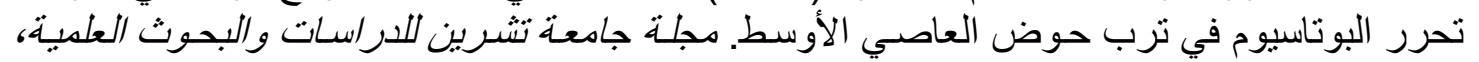

\section{سلسلة العلوم البيولوجية، 29 (1): 93- 110.}

Al- Semmak, Q and H, Abbas (2008). The Behavior Of Some Potassium Fertilizers In Cultivated Desert Soil Under Different Irrigation Systems. PhD Thesissubmitted to College of Agriculture, University of Baghdad, Iraq.

Al-Azawi, H. A. (2010). Effect of Cation Exchange Capacity On Availability Of Potassium To The Maize Crop In A Heavy Clay Soil. M.Sc. Thesis. Agronomy and Soil Science School of Environment and Rural Science. University of New England.

Al-Obaidi, M. A. and A. R. Hussain (2010). Kinetics of potassium adsorption and desorption in some Nineveh Governorate soils. Mesopotamia Agricultural Journal. 38 (4): 32 - 37.

Al-Wabel, M. A. ; Heil, D. M. ; Westfall, D. G. and K. A. Barbarick, (2002). Solution chemistry influence on metal mobility in biosolids - amended soils. Journal of Environment quality 31:1157 - 1165.

Anonymous, (2008). Key To Soil Taxonomy. Soil Survey Staff,10 ${ }^{\text {th }}$ Edition. USDA, NRCS. USA.

Badraoui, M. M. ; M. Agbani ; P. R. Bloom ; R. Bouabid ; B. Soudi ; A. Mimouni and S. Bouchara (1992). Chemistry and Mineralogy of Potassium. MoroccoInt. Centre Of Agricultural Researches In Dry Areas. ICARDA.

Bohn, H. L.; B. I. McNeal and G. A. Oconnor (2005). Soil Chemistry. John and Willy's sons. Inc. USA.

Carter, M. R. and E. G. Gregorich (2008). Soil Sampling and Methods of Analysis. $2^{\text {nd }}$ edition. Canadian Society of Soil Science. Canada.

Cracksi, T. H. and D. L. Sparks (1985). A modified miscible displacement technique for investigating adsorption-desorption kinetics in Soils. Soil Science Society Journal. 49:1114-1116.

$\mathrm{Du}$, Y. J. (2004). Some factors controlling the adsorption of potassium ions on clay soils. Applied Clay Sciences. 27: 209 - 213.

Ghosh, B. N. and R. D. Sing (2001). Potassium release characteristics for some soils of Uttar Pradesh hills varying in altitude and their clay mineralogy. Geoderma. 104: $134-144$.

Huang,. M. (2005). Chemistry of Potassium In Soil. American Society Of Soil Sciences. WI. p 227 - 292. 
Jackson, M. L. and L. X. Luo(1985).Potassium release on drying of soil nonexchangeable by protonation of mica. Soil Science 141:225-229.

Jalali, M. (2006). Site-Specific Potassium Application Based On The Fertilizer Potassium Availability Index Of Soil. Precision Agric. Springer Science Business Media, LL.C.

Karabachsch, M, and B. Ulrich (2007). Kalium - dynamic and kalium - fixiegrung, Nordwesticaischer Boden. Zeitschrift Für Pflanzenernahrung und Bodenkunde. 141 (5): 535 - 546.

Khodabaksh, P. K. (2006). Sorption - desorption behavior of phosphorous and potassium in four series of Isfahan - 18th World Congress Of Soil Science. July 9 - 15, 2005. Philadelphia, Pennsylvania, USA 154 - 159.

Kolahchi, Z. I. and M. Jalali (2007). Effect of water quantity on the leaching of potassium from sandy soil. Journal of Arid Environment. 68: 624 - 639.

Mam Rasul, Gh. A. (2008). Physio-Chemical Behavior Of Potassium In Predomination Soil Orders In Sulaimania Governorate. Ph.D Thesis Agriculture College, University of Sulaimania.

Mam Rasul, Gh. A. and M. A. Al-Obaidi (2011). Kinetics of potassium desorption from entisols, vertisols and mollisols using modified miscible displacement technique in Sulaimania Governorate. Mesopotamia Journal of Agriculture. 39 (3): $32-38$.

McLean, E. O. and M. E. Watson (1985). Soil measurements of plant available potassium:227 - 308 in R-D-Muson(ed). Potassium In Agriculture- ASA.CSSA. And SSA, Madison, W.I.

Poonia, S. R. and E. A. Nicderbudde (2007). Exchange equilibrium of potassium in soils. $\amalg-$ Effect of $\mathrm{K}+$ fertilization on $\mathrm{K}^{+}-\mathrm{Ca}^{2+}$ exchange. Journal of Plant Nutrition and Soil Science. 149 (0): 691 - 701.

Rao, Ch. S. ; A. Swarup ; A. S. Rao and U. R. Gobal (2004). Kinetics of non exchangeable potassium release from rapaquept as influenced by long term cropping fertilization and manuring. Australian Journal of Soil Researches. 37 (7): 317 - 328.

Richards, L. A. (1954). Diagnosis and Improvement Of Saline and Alkali Soils. Agriculture Handbook No.60 USDA Washington.

Rowell, D. L. and P. Dirmitories (2002). Diffusion and cation exchange during the reclamation of saline structured soils. Geoderma 107: 271 - 278.

Shanwall, A. V. (2006). Characterization of soil potassium derived from sorption desorption experiments. Plant and Soil. 251 (2): 331 - 341.

Shanwall, A. V. and S. S. Dahiya (2006). Potassium Dynamics and Mineralogy Encyclopedia of Soil Science. $2^{\text {nd }}$ edition. In lal (ed.).

Simard, R. R. ; G. R. Dekimpe and J. Zizka (1992). Release of potassium and magnesium from soil fraction and its kinetics, Soil Science Society of American Journal. 55: 1421 - 1429. 
Simonsson, M. ; S. Anderson ; Y. Angrich - Ranget ; S. Hillier ; L. Mattsson and I. Oborn (2007). Potassium release and fixation as function of fertilizer application rate and soil parent material. Geoderma 140: 188 -198.

Sparks, D.L. (2000). Bioavailability of soil potassium - In Handbook Of Soil. Walcolmn -Summered 2000 CORC Press. New York.

Sparks, D.L. (2003). Environmental Soil Chemistry of. $2^{\text {ed }}$ edition. Elevier Science (USA).

Wang, J. J.; D. L. Harvell and P. F. Bell (2004). Potassium buffering characteristics of three soils low in exchangeable potassium. Soil Science Society of American Journal. 68: 654 - 661. 
Mesopotamia J. of Agric.

Vol. (45) No. (2) 2017
ISSN: 2224 - 9796 (Online)

ISSN: 1815 - 316 X (Print)
مجلـــــة زر اعـــة الــر افديـنـ

المجلد (45) العدد (2) 2017 